International Mathematical Forum, 2, 2007, no. 36, 1767 - 1772

\title{
Interpolation of Triple-Banach Spaces on $R^{3}$
}

\author{
S.M.S. Modarres \\ Department of Mathematics \\ Yazd University \\ Yazd 89195-741, Iran \\ smodarres@yazduni.ac.ir \\ Z. Ghorbani \\ Department of Mathematics \\ Yazd University \\ Yazd 89195-741, Iran \\ ghorbani_aylin@yahoo.com
}

\begin{abstract}
Let $\bar{A}=\left(A_{0}, A_{1}, A_{2}\right)$ be a compatible triple of Banach spaces. We may define the interpolation method in $R^{3}$, and prove some related lemma and theorem.
\end{abstract}

Mathematics Subject Classification: 47A60,46M35

Keywords: interpolation space.

\section{Introduction}

Our main reference to the theory of interpolation space is [1]. Let $\bar{A}=$ $\left(A_{0}, A_{1}, A_{2}\right)$ be a compatible triple of Banach spaces, we define the space $\mathcal{F}(\bar{A})$ of all function $\mathrm{f}$ with values in $\sum(\bar{A})$, which are bounded and continuous on the $\bar{S}=\left\{x=\left(x_{0}, x_{1}, x_{2}\right) \in R^{3}: 0 \leq x_{0} \leq 1\right\}$ and analytic on the $S=\left\{x=\left(x_{0}, x_{1}, x_{2}\right) \in R^{3}: 0<x_{0}<1\right\}$. And moreover, the functions $t \longrightarrow f(0, j, t)(j=0,1)$ and $t \longrightarrow f(1,1, t)$ are continuous function from the real line into $A_{0}, A_{1}$ and $A_{2}$, which tend to zero as $|t| \longrightarrow \infty$. Clearly, $\mathcal{F}(\bar{A})$ is a vector space. We provided $\mathcal{F}$ with the norm

$$
\|f\|_{\mathcal{F}}=\max \left(\sup \|f(0,0, t)\|_{A_{0}}, \sup \|f(0,1, t)\|_{A_{1}}, \sup \|f(1,1, t)\|_{A_{2}}\right) .
$$




\section{Main result}

Lemma 2.1 The space $\mathcal{F}$ is a Banach space.

\section{Proof.}

Suppose that $\sum_{n}\left\|f_{n}\right\|_{\mathcal{F}}<\infty$. Since $f_{n}(x)$ is bounded in $\sum(\bar{A})$, we have

$$
\left\|f_{n}(x)\right\|_{\sum(\bar{A})} \leq \max \left(\sup \left\|f_{n}(0,0, t)\right\|_{\sum(\bar{A})}, \sup \left\|f_{n}(0,1, t)\right\|_{\sum(\bar{A})}, \sup \left\|f_{n}(1,1, t)\right\|_{\sum(\bar{A})}\right)
$$

Since $A_{j} \subset \sum(\bar{A})$, we conclude that

$$
\left\|f_{n}(x)\right\|_{\sum(\bar{A})} \leq\left\|f_{n}\right\|_{\mathcal{F}}
$$

Since $\sum(\bar{A})$ is a Banach space. it follows that $\sum_{n} f_{n}$ converges uniformly on $\mathrm{S}$ to a function $\mathrm{f}$ in $\sum(\bar{A})$. Thus $\mathrm{f}$ is bounded and continuous on $\mathrm{S}$ and analytic in $S_{0}$. Furthermore, $\left\|f_{n}(0, j, t)\right\|_{A_{j}} \leq\left\|f_{n}\right\|_{\mathcal{F}}(j=0,1)$ and $\left\|f_{n}(1,1, t)\right\|_{A_{2}} \leq\left\|f_{n}\right\|_{\mathcal{F}}$. Thus $\sum_{n} f_{n}(0, j, t)$ and $\sum_{n} f_{n}(1,1, t)$ converges uniformly in to a limit in $A_{j}$ and $A_{2}$, which must coincide with the limit in $\sum(\bar{A})$. Therefore, $f(0, j, t) \in$ $A_{j}, f(1,1, t) \in A_{2}$ and $\sum_{n} f_{n}(0, j, t), \quad \sum_{n} f_{n}(1,1, t)$ converges uniformly to $f(0, j, t)$ in $A_{j}$ and $f(1,1, t)$ in $A_{2}$. But then it follows that $f=\mathcal{F}$, and that $\sum_{n} f_{n}$ converges to $\mathrm{f}$ in $\mathcal{F}$.

We now define the interpolation functor $\bar{A}_{[\theta]}$. The space $\bar{A}_{[\theta]}$ consists of all $a \in \sum(\bar{A})$ such that $a=f(\theta)((\theta, 0,0)=\theta)$ for some $f \in \mathcal{F}(\bar{A})$. The norm on $\bar{A}_{[\theta]}$ is

$$
\|a\|_{[\theta]}=\inf \left\{\|f\|_{\mathcal{F}}: f(\theta)=a, f \in \mathcal{F}\right\} .
$$

Definition 2.2 The interpolation space $A$ and $B$ are exact of exponent $\theta$ and $\eta,(0 \leq \theta+\eta \leq 1)$ if

$$
\|T\|_{A, B} \leq\|T\|_{A_{0}, B_{0}}^{1-(\eta+\theta)}\|T\|_{A_{1}, B_{1}}^{\theta}\|T\|_{A_{2}, B_{2}}^{\eta} .
$$

Theorem 2.3 The space $\bar{A}_{[\theta]}$ is a Banach space. The functor $\bar{A}_{[\theta]}$ is an exact interpolation functor of exponent $\theta$ and $\eta$.

\section{Proof.}

The linear mapping $f \longrightarrow f(\theta)$ is a continuous mapping from $\mathcal{F}(\bar{A})$ to $\sum(\bar{A})$ since $\|f(\theta)\|_{\sum(\bar{A})} \leq\|f\|_{\mathcal{F}}$. The kernel of this mapping is $\mathcal{N}_{\theta}=\{f: f \in$ $\mathcal{F}, f(\theta)=0\}$. Clearly, $\bar{A}_{[\theta]}$ is isomorphic and isometric to the quotient space $\mathcal{F}(\bar{A}) / \mathcal{N}_{\theta}$. Since $\mathcal{N}_{\theta}$ is closed, it follows that $\bar{A}_{[\theta]}$ is a Banach space. Assume that $\mathrm{T}$ maps $A_{j}$ to $B_{j}$ with norm $M_{j}(j=0,1,2)$. Given $a \in \bar{A}_{[\theta]}$ and $\varepsilon>0$, there is a function $f \in \mathcal{F}$, such that $f(\theta)=a$ and $\|f\|_{\mathcal{F}} \leq\|a\|_{[\theta]}+\varepsilon$. Put $g(x)=M_{0}^{x-1} M_{1}^{-x+\eta} M_{2}^{-x+\theta} T(f(x))$. g belongs to the class $\mathcal{F}(\bar{B})$. Moreover, $\|g\|_{\mathcal{F}} \leq\|f\|_{\mathcal{F}} \leq\|a\|_{[\theta]}+\varepsilon$. But now $g(\theta+\eta)=M_{0}^{\theta+\eta-1} M_{1}^{-\theta} M_{2}^{-\eta} T(a)$ and hence 
we conclude that $\|T(a)\|_{[\theta]} \leq M_{0}^{1-\theta+\eta} M_{1}^{\theta} M_{2}^{\eta}\|g\|_{\mathcal{F}} \leq M_{0}^{1-\theta+\eta} M_{1}^{\theta} M_{2}^{\eta}\|a\|_{[\theta]}+\dot{\varepsilon}$, where $\dot{\varepsilon}=M_{0}^{1-\theta+\eta} M_{1}^{\theta} M_{2}^{\eta} \varepsilon$. This gives the result.

Now we may introduce a second interpolation method in $R^{3}$. This is based on a space $\tilde{\mathcal{F}}(\bar{A})$ of analytic functions, defined as follows. The functions $\mathrm{g}$ in $\tilde{\mathcal{F}}(\bar{A})$ are defined on $\mathrm{S}$ with values in $\sum(\bar{a})$. Moreover they have the following properties:

$(i)\|g\|_{\sum(\bar{A})} \leq c(1+|x|), \quad\left(|x|=\left(\sum_{i=0}^{i=2} x_{i}^{2}\right)^{1 / 2}\right)$

(ii) $\mathrm{g}$ is continuous on $\mathrm{S}$ and analytic on $S_{0}$,

(iii) $g\left(0, j, t_{1}\right)-g\left(0, j, t_{2}\right)$ has values in $A_{j}$ and $g\left(1,1, t_{1}\right)-g\left(1,1, t_{2}\right) \in A_{2}$ for all real values of $t_{1}$ and $t_{2}$ and for $j=0,1$, and

$$
\begin{aligned}
& \|g\|_{\tilde{\mathcal{F}}}=\max \left(\sup _{t_{1}, t_{2}}\left\|\frac{g\left(0,0, t_{1}\right)-g\left(0,0, t_{2}\right)}{t_{1}-t_{2}}\right\|_{A_{0}}\right. \\
& \left., \sup _{t_{1}, t_{2}}\left\|\frac{g\left(0,1, t_{1}\right)-g\left(0,1, t_{2}\right)}{t_{1}-t_{2}}\right\|_{A_{1}}, \sup _{t_{1}, t_{2}} \mid \frac{g\left(1,1, t_{1}\right)-g\left(1,1, t_{2}\right)}{t_{1}-t_{2}} \|_{A_{2}}\right) .
\end{aligned}
$$

is finite.

Lemma 2.4 The space $\tilde{\mathcal{F}}(\bar{A})$, reduced modulo constant functions and provided with the norm $\|g\|_{\tilde{\mathcal{F}}}$, is a Banach space.

Proof.

From the conditions it follows easily that if $h \neq 0$ is a real number then

$$
\left\|\frac{g(x+h)-g(x)}{h}\right\|_{\sum(\bar{A})} \leq\|g\|_{\tilde{\mathcal{F}}}(x \in S) .
$$

Thus we obtain

$$
\|\dot{g}(x)\|_{\sum(\bar{A})} \leq\|g\|_{\tilde{\mathcal{F}}}
$$

We therefore see that if $\|g\|_{\tilde{\mathcal{F}}}=0$ then $\mathrm{g}$ is constant. This implies that $\tilde{\mathcal{F}}$ modulo constants is a normed space. We also that $x \in S_{0}$

$$
\|g(x)-g(0)\|_{\sum(\bar{A})} \leq|x|\|g\|_{\tilde{\mathcal{F}}}
$$

Now suppose that $\sum_{n}\left\|g_{n}\right\|_{\tilde{\mathcal{F}}}<\infty$. Then $\sum_{n}\left(g_{n}(x)-g_{n}(0)\right)$ converges uniformly on every compact subset of $S_{0}$. The limit $g(x)$ satisfies (i) and (ii). Moreover it follows that the series $\sum_{n}\left(g_{n}\left(0, j, t_{1}\right)-g_{n}\left(0, j, t_{2}\right)\right)$ and $\sum_{n}\left(g_{n}\left(1,1, t_{1}\right)-\right.$ $\left.g_{n}\left(1,1, t_{2}\right)\right)$ converges in $A_{j}$ and $A_{2}$. Thus $g\left(0, j, t_{1}\right)-g\left(0, j, t_{2}\right) \in A_{j}, g\left(1,1, t_{1}\right)-$ $g\left(01,1, t_{2}\right) \in A_{2}$ and is the sum of the series $\sum_{n}\left(g_{n}\left(0, j, t_{1}\right)-g_{n}\left(\underset{\tilde{\mathcal{F}}}{0}, j, t_{2}\right)\right)$, $\sum_{n}\left(g_{n}\left(1,1, t_{1}\right)-g_{n}\left(1,1, t_{2}\right)\right)$ in $A_{j}$ and $A_{2}$. Therefore $g \in \tilde{\mathcal{F}}$, i.e. $\tilde{\mathcal{F}}$ is compact.

We now define the space $\bar{A}^{[\theta]}$ in the following way. For a given $\theta$ such that $0<\theta<1$ we let $\bar{A}^{[\theta]}$ cosist of all $a \in \sum(\bar{A})$ such that $a=\dot{g}(\theta)$ for some $g \in \tilde{\mathcal{F}}$. The norm on $\bar{A}^{[\theta]}$ is

$$
\|a\|^{[\theta]}=\inf \left\{\|g\|_{\tilde{\mathcal{F}}}: \dot{g}(\theta)=a, g \in \tilde{\mathcal{F}}\right\} .
$$


Theorem 2.5 The space $\bar{A}^{[\theta]}$ is a Banach space and functor $\bar{A}^{[\theta]}$ is an exact interpolation functor of exponent $\theta$ and $\eta$.

\section{Proof.}

Since $\|\dot{g}(\theta)\|_{\sum(\bar{A})} \leq\|g\|_{\tilde{\mathcal{F}}}$, we see that the mapping $g \longrightarrow g(\theta)$ from $\tilde{\mathcal{F}}$ into $\sum(\bar{A})$ is continuous. The kernel $\mathcal{N}^{\theta}$ of this mapping is closed and the range is $\bar{A}^{[\theta]}$. The norm on $\bar{A}^{[\theta]}$ is the quotient norm on $\tilde{\mathcal{F}} / \mathcal{N}^{\theta}$. Thus $\bar{A}^{[\theta]}$ is a Banach space.

assume that $T: A_{j} \longrightarrow B_{j}$ with norm $M_{j}$ for $j=0,1,2$. Then we chose a function $g \in \tilde{\mathcal{F}}$, such that $g(\theta)=a,\|g\|_{\tilde{\mathcal{F}}} \leq\|a\|^{[\theta]}+\varepsilon$. Consider the function

$$
\begin{gathered}
h(x)=\left[M_{0}^{k+\eta-1} M_{1}^{-k} M_{2}^{\theta-\eta-k} T(g(k))\right]_{k=0}^{k=x} \\
\int_{0}^{x} \log M_{0} / M_{1} M_{2} M_{0}^{k+\eta-1} M_{1}^{-k} M_{2}^{\theta-\eta-k} T d(g(k)) .
\end{gathered}
$$

The integral is taken along any path in $\mathrm{S}$ which connects 0 and $\mathrm{x}$. If the path has all its points in $S_{0}$ except 0 and possibly x we may integrate by parts. In fact, if $k \in S_{0}$ we have $d(T(g(k))) / d k=T(\dot{g}(k))$ and $g(k)$ is bounded and continuous on $S_{0}$. Thus $d(T(g(k))) / d k$ is continuous on $S_{0}$ and has bounded norm in $\sum(\bar{B})$. Thus we may integrate by parts, and we have obtain, for any path in $\mathrm{S}$,

$$
h(x)=\int_{0}^{x} M_{0}^{k+\eta-1} M_{1}^{-k} M_{2}^{\theta-\eta-k} T d(g(k)),
$$

where in general the integral is to be interpreted as a vector-valued Stieltjes integral. It follow that

$$
\|h(x)\|_{\sum(\bar{B})} \leq c|x| .
$$

Next we note that $T\left(g\left(0, j, t_{1}\right)-g\left(0, j, t_{2}\right)\right) \in B_{j}, g\left(1,1, t_{1}\right)-g\left(01,1, t_{2}\right) \in$ $B_{2}$ and is a Lipschitz function in $B_{j}$. Thus it follows that

$$
\left\|h\left(0, j, t_{1}\right)-h\left(0, j, t_{2}\right)\right\|_{B_{j}} \leq M_{j}^{-1} \int_{t_{1}}^{t_{2}}\|T(d g(0, j, t))\|_{B_{j}},
$$

and

$$
\left\|h\left(1,1, t_{1}\right)-h\left(1,1, t_{2}\right)\right\|_{B_{2}} \leq M_{2}^{-1} \int_{t_{1}}^{t_{2}}\|T(d g(1,1, t))\|_{B_{2}} .
$$

if $t_{1}<t_{2}$. But the right hand side is bounded by

$$
\left.\int_{t_{1}}^{t_{2}} \| d g(0, j, t)\right)\left\|_{A_{j}} \leq\left(t_{2}-t_{1}\right)\right\| g\left\|_{\|} g\right\|_{\tilde{\mathcal{F}}}
$$

and

$$
\left.\int_{t_{1}}^{t_{2}} \| d g(1,1, t)\right)\left\|_{A_{2}} \leq\left(t_{2}-t_{1}\right)\right\| g\left\|_{\|} g\right\|_{\tilde{\mathcal{F}}}
$$

It follows that

$$
\|h\|_{\|} g\left\|_{\tilde{\mathcal{F}}}(\bar{B}) \leq\right\| a \|^{[\theta]}+\varepsilon .
$$


Now

$$
\grave{h}(\theta)=M_{0}^{\theta+\eta-1} M_{1}^{-\theta} M_{2}^{-\eta}(d / d k T(g(k))) M_{0}^{\theta+\eta-1} M_{1}^{-\theta} M_{2}^{-\eta} T(a) .
$$

This proves that $T(a)=M_{0}^{\theta+\eta-1} M_{1}^{-\theta} M_{2}^{-\eta} \hat{h}(\theta) \in \bar{B}^{[\theta]}$, and that

$$
\|T(a)\|^{[\theta]} \leq M_{0}^{1-\theta+\eta} M_{1}^{\theta} M_{2}^{\eta}\|a\|_{[\theta]}+\dot{\varepsilon} .
$$

This gives the result.

Theorem 2.6 Let $\bar{A}$ be a compatible Banach triple and put

$$
X_{j}=\bar{A}_{\left[\theta_{j}\right]}\left(0 \leq \theta_{j} \leq 1 ; j=0,1,2\right)
$$

Assume that $\triangle(\bar{A})$ is dense in the spaces $A_{j}$ and $\triangle(\bar{X})$. Then

$$
\bar{A}_{[\theta]} \subset \bar{X}_{[\theta]} .
$$

where $\theta=(1-(\alpha+\beta)) \theta_{0}+\alpha \theta_{1}+\beta \theta_{2}$.

Proof.

First we show that $\|a\|_{\bar{X}_{[\alpha+\beta]}} \leq\|a\|_{\bar{A}_{[\theta]}}$ if $a \in \bar{A}_{[\theta]}$. Take $a \in \bar{A}_{[\theta]}$ then there exists a function $f \in \mathcal{F}(\bar{A})$, such that $f(\theta)=a$ and $\|f\|_{\mathcal{F}} \leq\|a\|_{\bar{A}_{[\theta]}}+\varepsilon, \varepsilon>0$ being arbitrary. Put $f_{1}(x)=f\left((1-x) \theta_{0}+x\left(\theta_{1}+\theta_{2}\right)-\alpha \theta_{2}-\beta \theta_{1}\right)$. Then $f_{1}(\alpha+\beta)=a$ and

$$
\left\|f_{1}\right\|_{\mathcal{F}(\bar{X})} \leq\|a\|_{\bar{A}_{[\theta]}}
$$

This gives $\|a\|_{\bar{X}_{[\theta]}} \leq\|a\|_{\bar{A}_{[\theta]}}$.

\section{References}

[1] N. Aroszajn, E. Gagliardo, Interpolation spaces and interpolation methods, Ann. Mat. Pura Appl, 68(1965),

[2] J. Bergh, and J. Lofstrom, Interpolation spaces. An introduction, Springer, Berlin, 1976.

[3] F. Cobos, L. E. Person, Real interpolation of compact operator between quasi-Banach spaces, Math. Scand, 82(1998), 138-160.

[4] F. Cobos, T. Kohn and J. Peetre, Schatten-von Neumann classof multilinear forms, Duke. Math. J, 65 (1992), 121-156.

[5] P. Grisvard, Interpolation non commutative, Math. Natur, 52(1972), 1115. 
[6] P. Kree, interpolation d'espaces vectoriels qui ne sont ni normes, ni complets, Applications, Ann. Inst. Fourier, 17 (1967), 137-174.

[7] H. Triebel, Interpolation Theory, Function spaces, Differential Operators, North-Holland, (1978).

Received: December 16, 2006 